\title{
Prevalence of Chronic Metabolic Comorbidities in Acute Pancreatitis and Its Impact on Early Gastrointestinal Symptoms during Hospitalization: A Prospective Cohort Study
}

\author{
Rachel Goodger Kanageswari Singaram Maxim S. Petrov
}

School of Medicine, University of Auckland, Auckland, New Zealand

\section{Keywords}

Acute pancreatitis - Diabetes mellitus - Obesity · Metabolic syndrome - Gastrointestinal symptoms · Patient-reported outcomes

\begin{abstract}
Background: The prevalence of chronic comorbidities is increasing worldwide, and this has been paralleled by a growing interest in how these comorbidities affect patients with acute pancreatitis. The aim was to investigate the associations between pre-existing diabetes mellitus, obesity, metabolic syndrome, and gastrointestinal symptoms during the early course of acute pancreatitis. Methods: This was a prospective cohort study of patients with a primary diagnosis of acute pancreatitis. Study groups were formed based on the presence of metabolic comorbidities (pre-existing diabetes mellitus, obesity, and metabolic syndrome). Patient-reported outcomes (nausea, bloating, and abdominal pain) were collected prospectively every $24 \mathrm{~h}$ (including weekends and public holidays) over the first $72 \mathrm{~h}$ of hospitalization. Results: A total of 183 consecutive patients were enrolled. Of them, 111 (61\%) had at least one major metabolic comorbidity. Patients with pre-existing diabetes mellitus and those with metabolic syndrome had worse nausea at 49-72 h of hospitalization ( $p=0.017$ and $p=0.012$, respectively), but not at
\end{abstract}

other time points. Bloating and abdominal pain did not differ between the study groupings throughout the study period. The studied patient-reported outcomes did not differ significantly between acute pancreatitis patients with and without obesity at any point in time. Conclusion: More than 3 out of 5 patients hospitalized for acute pancreatitis have at least one major chronic metabolic comorbidity. The presence of metabolic comorbidities does not considerably and consistently affect early gastrointestinal symptoms in patients with acute pancreatitis.

(C) 2021 The Author(s)

Published by S. Karger AG, Basel

\section{Introduction}

The prevalence of chronic metabolic comorbidities (such as diabetes mellitus, obesity, and metabolic syndrome) has been increasing relentlessly worldwide $[1,2]$. At the same time, the incidence of acute pancreatitis (AP) - one of the most common acute gastrointestinal diseases - is projected to markedly increase by 2050 [3-5]. Numerous studies have shown that the above comorbidities have an impact on "hard" clinical outcomes during the course of AP such as mortality, ICU admission, and severity of AP [6-13].

R.G. and K.S. contributed equally to this work.

Correspondence to:

Maxim S.Petrov, max.petrov@gmail.com

karger@karger.com www.karger.com/bmh

Karger $\stackrel{\text { ' }}{5}$

BOPEN ACCESS
C 2021 The Author(s).

Published by S. Karger AG, Basel

This is an Open Access article licensed under the Creative Commons Attribution-NonCommercial-4.0 International License (CC BY-NC) (http://www.karger.com/Services/OpenAccessLicense), applicable to the online version of the article only. Usage and distribution for commercial purposes requires written permission. 
By contrast, "soft" clinical outcomes (such as patientreported outcomes) have received only little attention in the AP setting $[14,15]$. Given that the gastrointestinal system is one of the most affected organ systems during the course of AP and taking into account that there are no readily available instrumental methods to monitor gastrointestinal function during AP [16-20], consideration of patient-reported outcomes focused on gastrointestinal symptoms is conceptually appealing. However, there is a paucity of studies on these outcomes in the AP setting. Furthermore, the associations between chronic metabolic comorbidities and gastrointestinal symptoms in AP have not previously been investigated [21].

The primary aim was to determine the prevalence of chronic metabolic comorbidities in a prospective cohort of consecutive patients with AP. The secondary aim was to investigate whether these comorbidities affect gastrointestinal symptoms in patients with AP.

\section{Methods}

\section{Study Design}

The study was a prospective cohort study conducted at Auckland City Hospital (New Zealand) as part of the PICTOR project. Patients were included in the study if they had a diagnosis of AP, were at least 18 years of age, and gave informed consent. Diagnosis of AP required at least 2 of the following 3 criteria to be met:

- Abdominal pain suggestive of AP;

- Serum amylase and/or pancreatic amylase and/or lipase at least 3 times the upper limit of normal (i.e., amylase $\geq 405 \mathrm{IU} / \mathrm{L}$, pancreatic amylase $\geq 159 \mathrm{IU} / \mathrm{L}$, and lipase $\geq 231 \mathrm{IU} / \mathrm{L}$ );

- Characteristic findings of AP on computed tomography (e.g., diffuse or segmental enlargement of the pancreas and/or peripancreatic necrosis and/or pancreatic necrosis).

Patients were excluded from the study if they had/were

- Chronic pancreatitis

- More than $96 \mathrm{~h}$ after onset of symptoms

- Post-endoscopic retrograde cholangiopancreatography pancreatitis

- Intraoperative diagnosis

- Pregnant or postpartum

- Malignancy

- Non-English speakers

- Cognitive impairment.

All patients were managed according to the standardized AP clinical care protocol [22].

\section{Study Groups}

Study groups were formed based on the following 3 comorbidities: pre-existing diabetes mellitus, obesity, and metabolic syndrome. Pre-existing diabetes mellitus was defined as documented fasting plasma glucose $\geq 7.0 \mathrm{mmol} / \mathrm{L}$ and/or glycated haemoglobin $\geq 48 \mathrm{mmol} / \mathrm{mol}$ and/or treatment with antidiabetic medications prior to hospitalization for AP. Obesity was defined by BMI (kg/ $\mathrm{m}^{2}$ ) according to the cutoff points recommended by the World
Table 1. Patient characteristics

\begin{tabular}{|c|c|}
\hline & $N=183$ \\
\hline Age, years ${ }^{1}$ & $49(36-68)$ \\
\hline \multicolumn{2}{|l|}{ Sex, $n(\%)$} \\
\hline Men & $90(49.2)$ \\
\hline Women & $93(50.8)$ \\
\hline \multicolumn{2}{|l|}{ Ethnicity, $n$ (\%) } \\
\hline European & $102(55.7)$ \\
\hline Maori/Pacific Islander & $40(21.9)$ \\
\hline Others & $41(22.4)$ \\
\hline \multicolumn{2}{|l|}{ Aetiology, $n(\%)$} \\
\hline Alcohol-related & $44(24.0)$ \\
\hline Biliary & $84(45.9)$ \\
\hline Others & $55(30.1)$ \\
\hline \multicolumn{2}{|l|}{ Severity } \\
\hline APACHE II score ${ }^{1}$ & $5.0(3-12)$ \\
\hline \multicolumn{2}{|c|}{ Metabolic comorbidities, $n(\%)$} \\
\hline Diabetes mellitus & $22(12)$ \\
\hline Obesity & $48(26)$ \\
\hline Metabolic syndrome & $102(56)$ \\
\hline
\end{tabular}

APACHE II, acute physiology and chronic health evaluation II. ${ }^{1}$ Data are presented as median (interquartile range).

Health Organization: normal $\left(18.5-24.9 \mathrm{~kg} / \mathrm{m}^{2}\right)$, overweight $(25-$ $\left.29.9 \mathrm{~kg} / \mathrm{m}^{2}\right)$, and obese $\left(\geq 30.0 \mathrm{~kg} / \mathrm{m}^{2}\right)$. Patients with a lower than normal BMI were excluded from the analysis. Weight and height were measured in the phase of convalescence in the present study (typically, 2 days prior to expected hospital discharge). Metabolic syndrome was defined according to the International Diabetes Federation definition [23]. This included central obesity (waist circumference $\geq 94 \mathrm{~cm}$ for European men and $\geq 80 \mathrm{~cm}$ for European women, with ethnicity-specific values for other ethnic groups) and at least 2 of the following 4 criteria: raised plasma fasting glucose (fasting plasma glucose $\geq 5.5 \mathrm{mmol} / \mathrm{L}$ or previously diagnosed type 2 diabetes mellitus), raised serum triglyceride level (triglyceride $\geq 1.7 \mathrm{mmol} / \mathrm{L}$ or specific treatment for dyslipidaemia), reduced high-density lipoprotein cholesterol (high-density lipoprotein cholesterol $<1.04 \mathrm{mmol} / \mathrm{L}$ for men and $<1.30 \mathrm{mmol} / \mathrm{L}$ for women), or arterial hypertension (systolic blood pressure $\geq 130 \mathrm{~mm} \mathrm{Hg}$ or diastolic blood pressure $\geq 85 \mathrm{~mm} \mathrm{Hg}$ or treatment of previously diagnosed hypertension) [23].

\section{Endpoints}

The study endpoints were nausea, abdominal pain, and bloating. For all recruited patients, the above endpoints were monitored every $24 \mathrm{~h}$ (including weekends and public holidays) for $72 \mathrm{~h}$ using a purposely designed AP diary. The response to each question was recorded on a Likert scale of $0-10(0-$ not at all and $10-$ most severe) in the AP diary.

\section{Statistical Analysis}

Data were analysed using SPSS Statistics for Windows version 25 (SPSS Inc., Chicago, IL, USA). The Shapiro-Wilk test was used to determine the normality of distribution of continuous variables. Where appropriate, the non-parametric Kruskal-Wallis H test, the 
Table 2. The relationship between metabolic comorbidities and gastrointestinal symptoms in the first $72 \mathrm{~h}$ of hospitalization for acute pancreatitis

\begin{tabular}{|c|c|c|c|c|c|c|c|c|c|c|c|}
\hline $\begin{array}{l}\text { Time } \\
\text { period }\end{array}$ & Symptom & \multicolumn{4}{|l|}{ Adiposity } & \multicolumn{3}{|c|}{ Diabetes mellitus } & \multicolumn{3}{|c|}{ Metabolic syndrome } \\
\hline \multirow{2}{*}{$0-24 \mathrm{~h}$} & Abdominal pain & $9.0(7.0,10.0)$ & $8.0(6.0,9.8)$ & $8.0(7.0,10.0)$ & 0.489 & $9.0(7.5,10.0)$ & $8.0(6.8,10.0)$ & 0.163 & $8.0(7.0,9.5)$ & $8.0(5.0,8.0)$ & 0.135 \\
\hline & Bloating & $3.5(0.0,7.0)$ & $5.0(0.8,7.0)$ & $5.0(0.0,7.0)$ & 0.407 & $4.5(0.0,5.3)$ & $5.0(0.0,7.0)$ & 0.219 & $5.0(0.0,7.0)$ & $5.0(0.0,7.0)$ & 0.890 \\
\hline $25-48 \mathrm{~h}$ & Bloating & $3.0(0.0,5.5)$ & $4.0(0.5,6.0)$ & $3.0(0.0,5.0)$ & 0.275 & $2.5(0.0,6.5)$ & $3.0(0.0,5.3)$ & 0.679 & $2.5(0.0,6.5)$ & $3.0(0.0,5.3)$ & 0.679 \\
\hline \multirow[t]{3}{*}{$49-72 \mathrm{~h}$} & Nausea & $0.0(0.0,4.0)$ & $0.5(0.0,3.5)$ & $0.0(0.0,2.5)$ & 0.405 & $2.5(0.3,6.5)$ & $0.0(0.0,3.0)$ & $0.017^{*}$ & $2.0(0.0,5.0)$ & $0.0(0.0,0.0)$ & $0.012^{*}$ \\
\hline & Abdominal pain & $3.0(1.0,6.0)$ & $4.5(3.0,7.0)$ & $5.0(2.0,6.0)$ & 0.230 & $5.0(2.5,8.0)$ & $4.0(2.0,6.0)$ & 0.376 & $5.0(2.0,7.0)$ & $3.0(1.5,4.5)$ & 0.074 \\
\hline & Bloating & $1.5(0.0,5.8)$ & $4.0(0.3,5.0)$ & $2.0(0.0,5.0)$ & 0.377 & $3.0(0.0,5.5)$ & $3.0(0.0,5.0)$ & 0.848 & $4.0(0.0,6.0)$ & $3.0(1.5,5.0)$ & 0.931 \\
\hline
\end{tabular}

Data are presented as median (interquartile range). ${ }^{*} p<0.05$

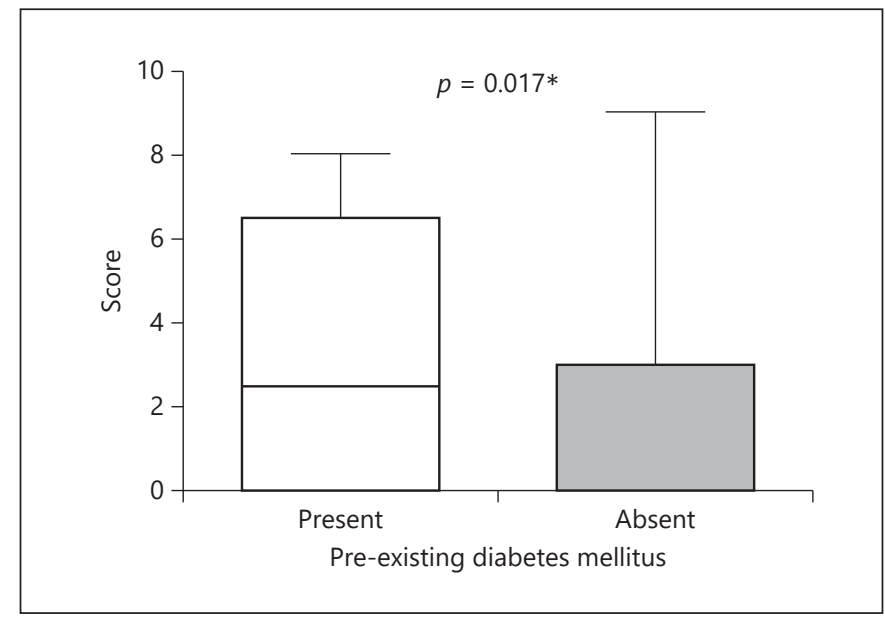

Fig. 1. Nausea scores at $49-72 \mathrm{~h}$ after hospitalization in acute pancreatitis patients with and without pre-existing diabetes mellitus.

$\chi^{2}$ test, and the Fisher's exact test were conducted. The potential confounding factors were then analysed using the Kruskal-Wallis $\mathrm{H}$ test to investigate their individual effect on each study outcome. All continuous data were presented as median and interquartile range (twenty-fifth and seventy-fifth percentiles). All categorical data were presented as absolute and relative frequencies. In all analyses, $p$ values $<0.05$ were accepted as statistically significant.

\section{Results}

\section{Characteristics of the Cohort}

A total of 183 consecutive patients with AP met the study eligibility criteria. These patients were admitted to the hospital in 12 (interquartile range 6-30) hours after onset of symptoms. Other characteristics are presented in Table 1. A total of 48 (26\%) patients were obese, $22(12 \%)$ patients had pre-existing diabetes mellitus, and $102(56 \%)$ had metabolic syndrome. Overall, 111 (61\%) patients in the study cohort had at least one major chronic metabolic comorbidity (obesity, pre-existing diabetes mellitus, or metabolic syndrome) at the time of hospitalization.

\section{Pre-Existing Diabetes Mellitus}

Comparison of baseline characteristics revealed a significant difference in age and APACHE II score between patients with and without pre-existing diabetes mellitus. The median age of those with and without pre-existing diabetes mellitus was 62 and 47 years, respectively ( $p=$ 0.010 ). The median APACHE II scores of those with and without pre-existing DM were 9 and 5 , respectively ( $p=$ 0.002 ). There were no differences between the groupings in terms of sex and ethnicity. The nausea score at 49-72 h of hospitalization differed significantly $(p=0.017)$ between patients with pre-existing DM and those without it (Fig. 1). There were no significant differences between the groupings at $0-24$ and $25-48 \mathrm{~h}$ of hospitalization. Abdominal pain and bloating did not differ between the study groupings throughout the study period (Table 2).

\section{Obesity}

Comparison of baseline characteristics revealed a significant difference in terms of ethnicity between the BMI categories. Patients of the Maori/Pacific Island origin had a greater prevalence of obesity (58\%) compared with those of European or other origin (17\% and 20\%, respec- 


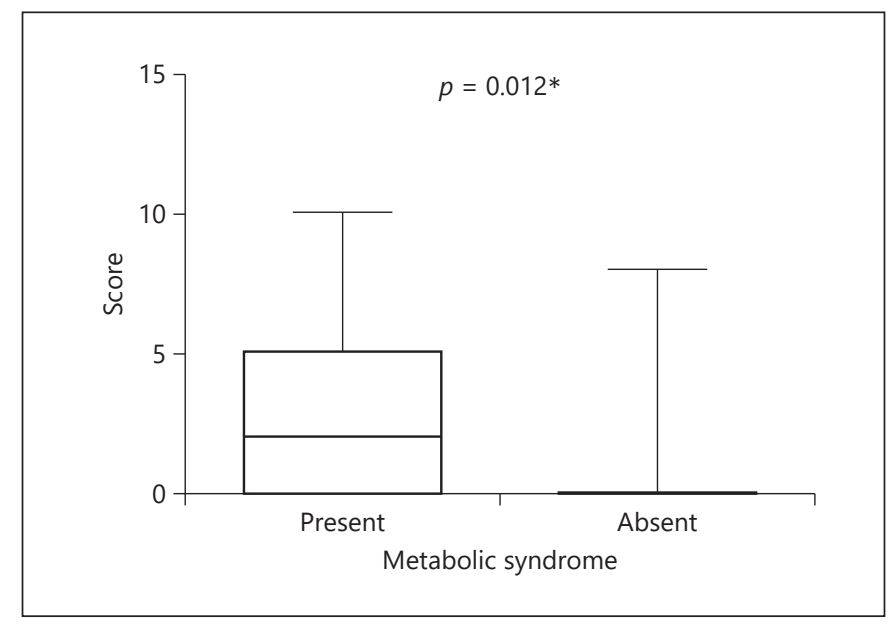

Fig. 2. Nausea scores at $49-72 \mathrm{~h}$ after hospitalization in acute pancreatitis patients with and without metabolic syndrome.

tively) ( $p<0.001)$. There were no significant differences between the groupings in terms of age, sex, and APACHE II score. There were no significant differences between the groupings at $0-24,25-48$, and $49-72 \mathrm{~h}$ of hospitalization (Table 2).

\section{Metabolic Syndrome}

Comparison of baseline characteristics revealed a significant difference in terms of age between patients with and without metabolic syndrome. The median ages of those with and without metabolic syndrome were 50 and 37 years, respectively $(p=0.003)$. There were no differences between the groups in terms of sex, ethnicity, and APACHE II score. The nausea score at $49-72 \mathrm{~h}$ of hospitalization differed significantly $(p=0.012)$ between patients with metabolic syndrome and those without (Fig. 2). For the abdominal pain score, the prespecified threshold for statistical significance was just missed ( $p=$ 0.074 ) (Table 2). The bloating score did not differ significantly between the groupings at $49-72 \mathrm{~h}$ of hospitalization. There were no significant differences between the groupings at $0-24$ and $25-48 \mathrm{~h}$ of hospitalization (Table 2).

\section{Discussion}

This was the first prospective cohort study to explore the relationship between pre-existing chronic metabolic comorbidities and gastrointestinal symptoms in patients with AP. One novel aspect of the present study is that it investigated in a prospective fashion not a single comorbidity but a suite of chronic metabolic comorbidities and showed that more than 3 out of 5 patients hospitalized for AP have at least one major chronic metabolic comorbidity at the time of hospitalization. This high prevalence of chronic metabolic comorbidities did not translate into a high incidence of hypertriglyceridaemia-induced pancreatitis as only 4 (2.2\%) patients in out cohort had serum triglyceride levels of $>1,000 \mathrm{mg} / \mathrm{dL}$. The prevalence of individual comorbidities identified in the present prospective cohort study was broadly in agreement with previously published studies. In the present study, the prevalence of pre-existing diabetes mellitus was $12 \%$, which is similar to the findings from a retrospective cohort study from Japan that found that $11 \%$ of AP patients had preexisting diabetes mellitus [24]. Some other retrospective studies reported slightly higher prevalence of pre-existing diabetes mellitus in AP patients: $18 \%$ in a cohort study from Pennsylvania [12], $17 \%$ in a cohort study from California [25], and $19 \%$ in a cohort study from Taiwan [11]. In the present study, $26 \%$ of patients with AP were obese, which is in agreement with previously published prospective cohort studies: a prevalence of $24 \%$ in a study from India and $22 \%$ in a study from Belgium (all consistently using the same threshold of BMI $\geq 30 \mathrm{~kg} / \mathrm{m}^{2}$ to define obesity) $[6,10]$. The present study also found that $56 \%$ of patients with AP fulfilled the criteria for metabolic syndrome using the International Diabetes Federation definition, which is consistent with the findings of a prospective cohort study from Saudi Arabia demonstrating that $63 \%$ of AP patients met the same criteria [13].

Another novel finding of this clinical study is that patients with pre-existing diabetes mellitus and/or metabolic syndrome were significantly associated with worse nausea at $49-72 \mathrm{~h}$ of hospitalization, when compared with patients who did not have these comorbidities. Although the effect of pre-existing diabetes mellitus on gastrointestinal symptoms in patients with AP has not previously been investigated, the association between diabetes mellitus and gastrointestinal symptoms is well established [26]. A large population-based study of 15,000 adults showed that diabetes mellitus (when compared with health) was associated with an increased prevalence of upper and lower gastrointestinal symptoms such as nausea, abdominal distension, and gastroesophageal reflux [27]. A smaller study found that the prevalence of gastrointestinal symptoms such as diarrhoea $(p=0.030)$ and bloating $(p=0.038)$ was greater in patients with long-standing type 2 diabetes mellitus com- 
pared with controls without diabetes [28]. However, unlike in the present study, no significant difference was observed for nausea and vomiting between individuals with and without pre-existing diabetes mellitus ( $p=$ 0.267 ) [28]. This may be attributed to the difference in the study populations: acute setting patients hospitalized for AP versus community-based individuals with diabetes mellitus [28]. It is conceivable that more severe nausea in patients with AP who had pre-existing diabetes mellitus may be attributed to delayed gastric emptying, similar to that seen in diabetic patients $[29,30]$. However, whether or not the reported observation is due to diabetic gastroparesis cannot be concluded from the present study as no significant differences were observed in terms of other gastrointestinal symptoms (such as abdominal pain or bloating), and gastrointestinal motility was not measured in the present study. Furthermore, a type II error cannot be ruled out.

This study had several limitations that need to be acknowledged. The study population was limited to patients recruited from a single hospital. A multicentre study may provide more robust and accurate estimates of the relationship between various comorbidities and gastrointestinal symptoms in patients with AP. In particular, the present study included only a few patients with necrotizing pancreatitis $(n=12)$, and therefore it was not positioned well to investigate the studied associations in this specific subgroup of patients. Also, APACHE II scores were only significantly different in patients with diabetes (but not other metabolic comorbidities), possibly reflecting the predominantly mild course of AP in the study cohort. The non-normal distribution of the data meant that a non-parametric statistical analysis was chosen (as opposed to a parametric statistical test). A limitation of all non-parametric tests is that it is challenging to undertake flexible modelling (such as accounting for possible confounders). This was partially overcome by summarizing all possible confounders individually after each Kruskal-Wallis test. The findings in the present study were based on patient-reported assessment of gastrointestinal symptoms, and the results might have been influenced by patient misinterpretation at the time of assessment. This effect was mitigated by members of the research team explaining and recording the results of the questionnaire in person in the daily diary; therefore, patient misunderstanding would have had a minimal impact on the results. Furthermore, patients with a cognitive impairment and non-English speakers were excluded from the study a priori to minimize patient misinterpretation of the questionnaire. Also, the questionnaire used to assess gastrointestinal symptoms has not been previously validated. However, the usefulness and validity of a Likert scale for investigating similar outcomes such as gastrointestinal motility has been shown to be reliable and valid. Patient-reported symptoms and health outcomes are an important part of clinical studies, and the Likert scale is simple, intuitive, and easy to interpret by patients and researchers alike.

\section{Conclusion}

The present prospective cohort study found that $61 \%$ of patients with AP have at least one chronic metabolic comorbidity at the time of hospitalization. The presence of chronic metabolic comorbidities in the majority of AP patients highlights the complexity of AP care required, which may involve not only surgeons and gastroenterologists but also other healthcare professionals [5]. It is important that future research is focused on developing a better understanding of how metabolic comorbidities, alone or combined, affect clinical outcomes in patients with AP and the risk of new-onset metabolic sequelae of AP that develop after hospital discharge [31-36].

\section{Acknowledgment}

This study was part of the Clinical and epidemiOlogical inveStigations in Metabolism, nutritiOn, and pancreatic diseaseS (COSMOS) program.

\section{Statement of Ethics}

The study was approved by the Health and Disability Ethics Committee (NTX/12/06/051). All participants provided their written informed consent.

\section{Conflict of Interest Statement}

The authors have no conflicts of interest.

\section{Funding Sources}

This study was supported by the Health Research Council of New Zealand (grant 15/035 to Professor Max Petrov), which played no role in the study design; collection, analysis, or interpretation of data; or writing of the manuscript. 


\section{Author Contributions}

M.S.P. contributed to conceptualization and study design. R.G. and K.S. contributed to patient recruitment. R.G. and K.S. contributed to data acquisition. R.G. and K.S. contributed to analysis and interpretation of data. R.G. and K.S. contributed to statistical analysis. R.G. and K.S. drafted the manuscript. M.S.P. contributed to study supervision.

\section{Data Availability Statement}

All data generated or analysed during this study are included in this manuscript.

\section{References}

1 Danaei G, Finucane MM, Lu Y, Singh GM, Cowan MJ, Paciorek CJ, et al. National, regional, and global trends in fasting plasma glucose and diabetes prevalence since 1980: systematic analysis of health examination surveys and epidemiological studies with 370 country-years and 2.7 million participants. Lancet. 2011;378(9785):31-40.

2 Alberti K, Zimmet P, Shaw J. Metabolic syndrome-a new world-wide definition. a consensus statement from the international diabetes federation. Diabetic Med. 2006;23(5): $469-80$.

3 Xiao AY, Tan ML, Wu LM, Asrani VM, Windsor JA, Yadav D, et al. Global incidence and mortality of pancreatic diseases: a systematic review, meta-analysis, and meta-regression of population-based cohort studies. Lancet Gastroenterol Hepatol. 2016;1:45-55.

4 Cho J, Petrov MS. Pancreatitis, pancreatic cancer, and their metabolic sequelae: projected burden to 2050. Clin Transl Gastroenterol. 2020;11(11):e00251.

5 Petrov MS, Yadav D. Global epidemiology and holistic prevention of pancreatitis. Nat Rev Gastroenterol Hepatol. 2019;16(3):17584.

6 Thandassery RB, Appasani S, Yadav TD, Dutta U, Indrajit A, Singh K, et al. Implementation of the Asia-pacific guidelines of obesity classification on the APACHE-O scoring system and its role in the prediction of outcomes of acute pancreatitis: a study from India. Dig Dis Sci. 2014;59(6):1316-21.

7 Sempere L, Martinez J, de Madaria E, Lozano B, Sanchez-Paya J, Jover R, et al. Obesity and fat distribution imply a greater systemic inflammatory response and a worse prognosis in acute pancreatitis. Pancreatology. 2008; 8(3):257-64.

8 O'Leary D, O’Neill D, McLaughlin P, O'Neill S, Myers E, Maher M, et al. Effects of abdominal fat distribution parameters on severity of acute pancreatitis. World J Surg. 2012;36(7): 1679-85.

9 Yashima $\mathrm{Y}$, Isayama H, Tsujino T, Nagano R, Yamamoto K, Mizuno S, et al. A large volume of visceral adipose tissue leads to severe acute pancreatitis. J Gastroenterol. 2011;46(10): $1213-8$.
10 De Waele B, Vanmierlo B, Van Nieuwenhove Y, Delvaux G. Impact of body overweight and class I, II and III obesity on the outcome of acute biliary pancreatitis. Pancreas. 2006; 32(4):343-5.

11 Shen HN, Lu CL, Li CY. Effect of diabetes on severity and hospital mortality in patients with acute pancreatitis: a national population-based study. Diabetes Care. 2012;35(5): 1061-6.

12 Nawaz H, O'Connell M, Papachristou GI, Yadav D. Severity and natural history of acute pancreatitis in diabetic patients. Pancreatology. 2015;15(3):247-52.

13 Sawalhi S, Al-Maramhy H, Abdelrahman AI, Allah SE, Al-Jubori S. Does the presence of obesity and/or metabolic syndrome affect the course of acute pancreatitis?: A prospective study. Pancreas. 2014;43(4):565-70.

14 de-Madaria E, Sánchez-Marin C, Carrillo I, Vege SS, Chooklin S, Bilyak A, et al. Design and validation of a patient-reported outcome measure scale in acute pancreatitis: the PANPROMISE study. Gut. 2021;70(1):139-47.

15 Pendharkar SA, Petrov MS. Bringing patientcentered care to the fore in diseases of the pancreas. Gastroenterol Res Pract. 2015;2015: 459214.

16 Petrov M. Nutrition, inflammation, and acute pancreatitis. ISRN Inflamm. 2013;2013: 341410.

17 Petrov MS. Gastric feeding and "gut rousing" in acute pancreatitis. Nutr Clin Pract. 2014; 29(3):287-90.

18 Bevan MG, Asrani VM, Pendharkar SA, Goodger RL, Windsor JA, Petrov MS. Nomogram for predicting oral feeding intolerance in patients with acute pancreatitis. Nutrition. 2017;36:41-5.

19 Bevan MG, Asrani VM, Bharmal S, Wu LM, Windsor JA, Petrov MS. Incidence and predictors of oral feeding intolerance in acute pancreatitis: a systematic review, meta-analysis, and meta-regression. Clin Nutr. 2017; 36(3):722-9.

20 Pendharkar SA, Plank LD, Windsor JA, Petrov MS. Quality of life in a randomized trial of nasogastric tube feeding in acute pancreatitis. JPEN J Parenter Enteral Nutr. 2016; 40(5):693-8.
21 Goodger RL, Asrani VM, Windsor JA, Petrov MS. Impact of metabolic comorbidities on outcomes of patients with acute pancreatitis: a scoping review. Panminerva Med. 2016; 58(1):86-93.

22 Stigliano S, Sternby H, de Madaria E, Capurso G, Petrov MS. Early management of acute pancreatitis: a review of the best evidence. Dig Liver Dis. 2017;49(6):585-94.

23 Alberti K, Zimmet P, Shaw J. Metabolic syndrome - a new world-wide definition. A consensus statement from the international diabetes federation. Diabetic Med. 2006;23(5): 469-80.

24 Satoh K, Shimosegawa T, Masamune A, Hirota M, Kikuta K, Kihara Y, et al. Nationwide epidemiological survey of acute pancreatitis in Japan. Pancreas. 2011;40(4):503-7.

25 Frey C, Zhou H, Harvey D, White RH. Comorbidity is a strong predictor of early death and multi-organ system failure among patients with acute pancreatitis. J Gastrointest Surg. 2007;11(6):733-42.

26 Sadiya A. Nutritional therapy for the management of diabetic gastroparesis: clinical review. Diabetes Metab Syndr Obes. 2012;5:329-35.

27 Bytzer P, Talley NJ, Leemon M, Young LJ, Jones MP, Horowitz M. Prevalence of gastrointestinal symptoms associated with diabetes mellitus: a population-based survey of 15000 adults. Arch Intern Med. 2001;161(16):1989_ 96.

28 de Kort S, Kruimel JW, Sels JP, Arts IC, Schaper NC, Masclee AA. Gastrointestinal symptoms in diabetes mellitus, and their relation to anxiety and depression. Diabetes Res Clin Pract. 2012;96(2):248-55.

29 Samsom M, Vermeijden JR, Smout AJ, Van Doorn E, Roelofs J, Van Dam PS, et al. Prevalence of delayed gastric emptying in diabetic patients and relationship to dyspeptic symptoms: a prospective study in unselected diabetic patients. Diabetes Care. 2003;26(11): 3116-22.

30 Laway BA, Malik TS, Khan SH, Rather TA. Prevalence of abnormal gastric emptying in asymptomatic women with newly detected diabetes and its reversibility after glycemic control-a prospective case control study. J Diabetes Complications. 2013;27(1):78-81. 
31 Petrov MS. Post-pancreatitis diabetes mellitus: investigational drugs in preclinical and clinical development and therapeutic implications. Expert Opin Investig Drugs. 2021; 30(7):737-47.

32 Kimita W, Petrov MS. Iron metabolism and the exocrine pancreas. Clin Chim Acta. 2020; $511: 167-76$
33 Bharmal SH, Cho J, Alarcon Ramos GC, Ko J, Stuart CE, Modesto AE, et al. Trajectories of glycaemia following acute pancreatitis: a prospective longitudinal cohort study with 24 months follow-up. J Gastroenterol. 2020; 55(8):775-88

34 Cho J, Dalbeth N, Petrov MS. Bidirectional relationship between gout and diabetes mellitus in individuals after acute pancreatitis: a nationwide cohort study. J Rheumatol. 2020; 47(6):917-23.
35 Petrov MS. Metabolic trifecta after pancreatitis: exocrine pancreatic dysfunction, altered gut microbiota, and new-onset diabetes. Clin Transl Gastroenterol. 2019;10(10):e00086.

36 Petrov MS. Post-pancreatitis diabetes mellitus: prime time for secondary disease. Eur J Endocrinol. 2021;184(4):R137-R149. 